\title{
ESTUDO DO EFEITO DA PERMEABILIDADE NA PERFORMANCE DO HIDROCICLONE CILÍNDRICO FILTRANTE
}

\author{
F. F. SALVADOR ${ }^{1}$, Y. N. KYRIAKIDIS ${ }^{1}$, S. M. GONÇALVES ${ }^{1}$, M. A. S. BARROZO ${ }^{1}$, L. G. M. \\ VIEIRA $^{1 *}$ \\ ${ }^{1}$ Universidade Federal de Uberlândia, Faculdade de Engenharia Química \\ *e-mail: luizgustavo.ufu@gmail.com
}

\begin{abstract}
RESUMO
Os hidrociclones são equipamentos utilizados em diversos setores industriais e se baseiam no princípio da sedimentação centrífuga. Podem ser empregados em processos de classificação de partículas de acordo com tamanho/densidade, espessamento, deslamagem, retirada de gotas de óleo do líquido de interesse. Propostas para a modificação desse equipamento visando alavancar seu desempenho sempre estiveram presentes na literatura. Dessa maneira, o intuito deste trabalho foi dar continuidade aos estudos realizados pelo grupo de pesquisa da Faculdade de Engenharia Química da Universidade Federal de Uberlândia ao incorporar uma parede cilíndrica permeável, dando origem ao hidrociclone cilíndrico filtrante. Objetivou-se avaliar como a permeabilidade da matriz porosa cilíndrica influenciava o desempenho do hidrociclone $\mathrm{HCiF}$. Os experimentos foram realizados utilizando três permeabilidades distintas para a parede porosa. Os resultados mostraram que menores permeabilidades fornecem equipamentos com maior capacidade de processamento. $\mathrm{O}$ consumo energético foi, em média, 24\% menor no $\mathrm{HCiF}$ de menor permeabilidade se comparado ao de maior permeabilidade. A razão de líquido e a eficiência total de separação sofreram pequenas modificações devido ao uso de diferentes permeabilidades para a parede filtrante.
\end{abstract}

\section{INTRODUÇÃO}

O hidrociclone foi patenteado pela primeira vez em 1981 e, posteriormente, tornou-se um importante processo unitário na separação de partículas na indústria de mineração (CHINÉ; CONCHA, 2000). Esses equipamentos foram pouco utilizados durante algumas décadas, somente a partir de 1940 teve início a fabricação desse separador com tecnologias avançadas (SVAROVSKY, 1984). Desde então, vários hidrociclones foram instalados e hoje são considerados essenciais em muitas empresas. Os hidrociclones possuem vasta aplicação na área de mineração, siderúrgica, papel e celulose, alimentícia, entre outras.
Um hidrociclone consiste basicamente de uma seção cilíndrica conectada a uma seção cônica que possui, em seu vértice, uma abertura denominada de orifício de underflow. A alimentação é introduzida tangencialmente na parte cilíndrica, na qual há um tubo coaxial denominado vortex finder, pelo qual é descarregado o overflow (CARRISSO; CORREIA, 2004).

A separação em hidrociclones é realizada através da sedimentação centrífuga. Ao ser alimentada no hidrociclone a suspensão gera um vortex descente o que faz com que as partículas de maior densidade relativa sejam projetadas contra a parede e arrastadas até a saída inferior do mesmo (underflow). Já as partículas menores são 
arrastadas para o centro do equipamento formando um vortex ascendente, saindo pelo orifício de overflow (SVAROVSKY, 2000).

Maior eficiência na separação de sólidos suspensos, capacidade elevada em relação ao seu volume e área ocupada, controle operacional relativamente simples e baixo custo de investimento são vantagens apresentadas pelos hidrociclones quando comparados a outros equipamentos que possuem a mesma finalidade (SRIPRIYA et al., 2007).

A Figura 1 apresenta um esquema geral de um hidrociclone convencional com suas dimensões características.

Figura 1 - Representação esquemática de um hidrociclone com suas dimensões características

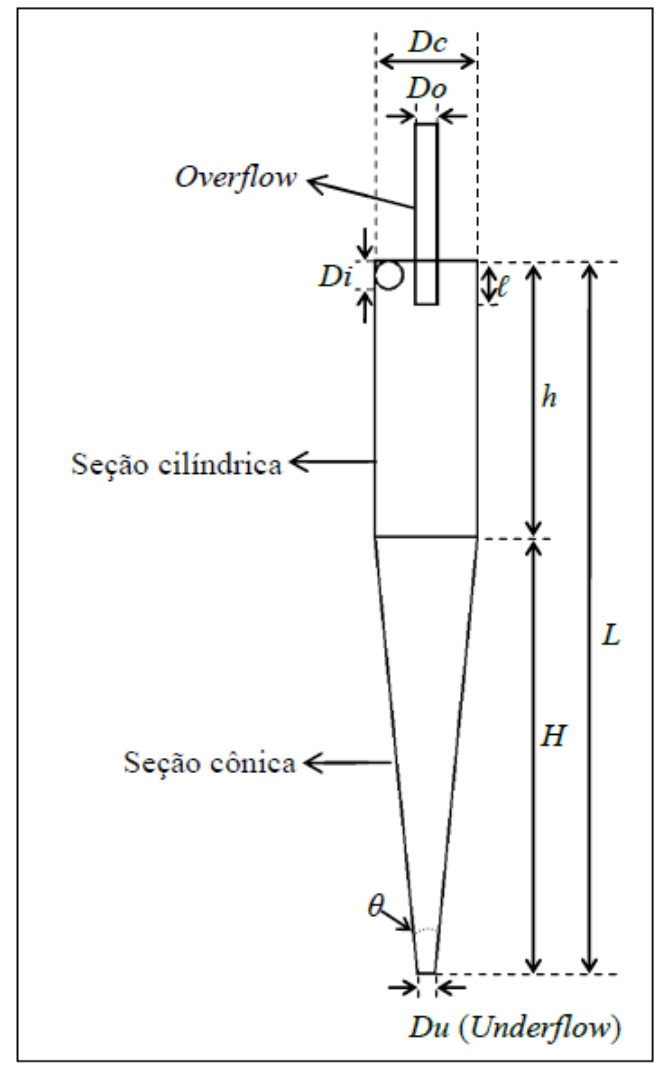

Dependendo das relações geométricas entre as principais dimensões de um tipo de hidrociclone é possível adequá-lo à execução de diversas atividades industriais. Essas configurações são denominadas famílias. A necessidade de um separador com alto poder classificador ou com grande poder concentrador é que determina o emprego de uma família específica em um processo.

Diferentes configurações geométricas e alterações na estrutura original do equipamento têm sido propostas, a fim de melhor adequá-lo a processos específicos (SILVA et al., 2012; HE et al., 2013; SILVA et al., 2015; DONG et al., 2013; GHODRAT et al., 2014).

Nesse quadro de idealização de um hidrociclone não convencional está inserido o hidrociclone cônico filtrante, uma nova configuração capaz de proporcionar melhor desempenho na separação sólido-líquido em relação a um hidrociclone convencional de mesma geometria e operando nas mesmas condições (SOUZA et al., 2000).

Segundo Vieira et al. (2007), o processo de separação do hidrociclone filtrante é similar ao do equipamento convencional. Sendo que no novo equipamento existe uma corrente adicional de fluido oriunda da região cônica porosa do separador além das correntes de alimentação, overflow e underflow.

Vieira et al. (2010) e Vieira et al. (2011) estudaram 25 hidrociclones cônico filtrantes com diferentes geometrias escolhidas através da técnica de planejamento de experimentos. Além disso, técnicas de fluidodinâmica computacional foram utilizadas para simular o escoamento interno nos hidrociclones e explicar fenomenologicamente os resultados obtidos nos testes experimentais. Dentre os 25 hidrociclones estudados, o de número 11 na matriz do planejamento (denominado H11) apresentou a melhor performance conciliando um baixo custo energético com uma alta eficiência de separação.

Nos estudos anteriores, foi verificado que os benefícios técnicos e científicos alcançados com o novo hidrociclone são inquestionáveis. Entretanto, acreditando que há possibilidade de melhorar ainda mais esse equipamento separador H11, o objetivo desse 
trabalho foi estudar uma nova configuração de hidrociclone filtrante, agora com uma parede cilíndrica porosa e avaliar a influência que distintas permeabilidades teriam sobre $\mathbf{o}$ desempenho do hidrociclone cilíndrico filtrante.

\section{MATERIAIS E MÉTODOS}

\subsection{Materiais}

O material utilizado neste trabalho foi a rocha fosfática, uma matéria-prima essencial na indústria de fertilizantes fosfatados, que tem grande importância econômica na região próxima ao município de Uberlândia. A densidade do material foi determinada por técnica de picnometria por deslocamento de gás hélio, realizada no equipamento AccuPyc 1330 da Micromeritics, e foi equivalente à $3245 \pm 1 \mathrm{~kg} / \mathrm{m}^{3}$.

A distribuição granulométrica da rocha fosfática foi obtida através de um equipamento analisador de partículas por difração a raios laser da Malvern modelo Mastersizer Microplus. O modelo RosinRammler-Bennet (RRB), representado pela Equação 1, foi o modelo que melhor se ajustou aos dados experimentais. Os parâmetros estimados do modelo foram $d_{63,2}=$ $12,15 \mu \mathrm{m}$ e $n=0,73$, com um coeficiente de correlação linear igual a 0,9927 .

$$
X=1-\exp \left[-\left(\frac{d_{p}}{d_{63,2}}\right)^{n}\right]
$$

A unidade experimental (Figura 2) usada neste trabalho era constituída de um tanque para armazenamento da suspensão de 0,25 $\mathrm{m}^{3}$; um agitador mecânico para homogeneizar a suspensão de $1,1 \mathrm{~kW}$ de potência que operava a $1600 \mathrm{rpm}$; uma bomba centrífuga $(3,7 \mathrm{~kW})$; um medidor de vazão do tipo Coriolis, cuja faixa de operação era de 0,0833 a $0,5000 \mathrm{~kg} / \mathrm{s}$; um manômetro digital
(0 a 700kPa); e um hidrociclone convencional (HCon) ou filtrante (HCiF).

Figura 2 - Diagrama esquemático da unidade experimental: 1-hidrociclone; 2-reservatório; 3bomba centrífuga; 4-válvula de bypass; 5-válvula; 6-medidor de vazão; 7-manômetro; 8-agitador mecânico.

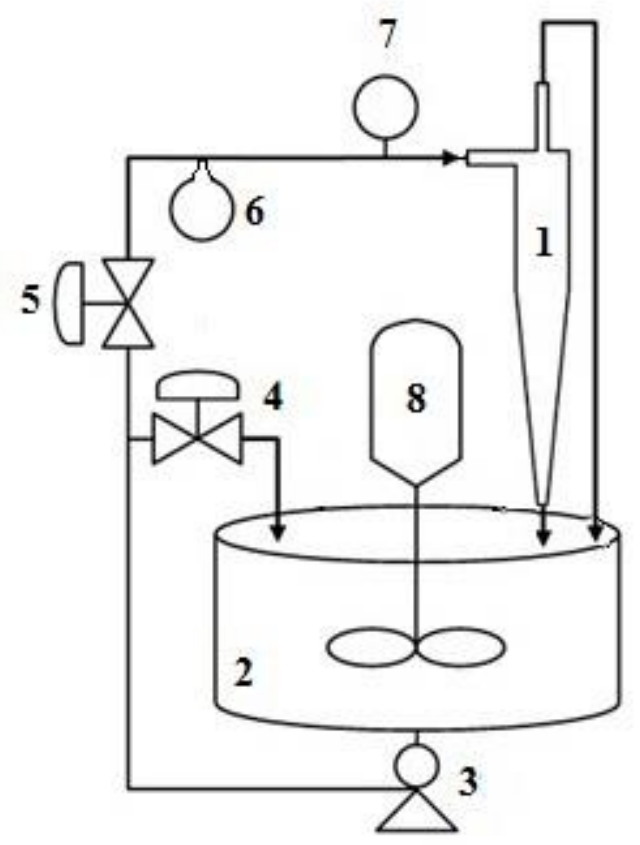

Conforme mencionado anteriormente, o objetivo deste trabalho foi avaliar presença de uma seção filtrante no hidrociclone e também a influência que distintas permeabilidades teriam sobre o desempenho do hidrociclone de geometria otimizada por Vieira et al. (2010). Dessa forma, as partes maciças de cada hidrociclone foram confeccionadas em latão e as seções filtrantes cilíndricas foram manufaturadas de partículas de bronze sinterizado. As principais relações geométricas desse hidrociclone são apresentadas na Tabela 1.

Para todos os equipamentos, o diâmetro da seção cilíndrica, o diâmetro do orifício de underflow e comprimento do duto de vortex finder foram, respectivamente, iguais a $30,3 \mathrm{e}$ $12 \mathrm{~mm}$. 
Tabela 1 - Relações geométricas dos hidrociclones convencional e filtrantes otimizadas por Vieira et al. (2010) e utilizadas neste trabalho.

\begin{tabular}{c|c}
\hline$D_{i} / D_{c}$ & 0,26 \\
$D_{o} / D_{c}$ & 0,22 \\
$L_{c} D_{c}$ & 6,90 \\
$\theta$ & $11,2^{\circ}$ \\
\hline
\end{tabular}

Para analisar o efeito da permeabilidade foi necessário o uso de cilindros porosos de granulometrias distintas. As matrizes porosas utilizadas no presente trabalho eram limpas e, portanto, foi necessário o procedimento de colmatação antes de utilizá-las nos ensaios. Após este procedimento, foram realizados testes de filtração conforme Rocha e Vieira (2012) para a determinação das permeabilidades das paredes filtrantes. Os valores obtidos para tal propriedade estão especificados na Tabela 2.

Tabela 2 - Propriedades das matrizes porosas cilíndricas.

\begin{tabular}{cc}
\hline Equipamento & Permeabilidade $\left(\mathrm{m}^{2}\right)$ \\
\hline HCiF-k1 & $1,91.10^{-16}$ \\
HCiF-k2 & $2,94.10^{-16}$ \\
HCiF-k3 & $8,85.10^{-16}$ \\
\hline
\end{tabular}

\subsection{Metodologia Experimental}

Após a escolha do tipo de hidrociclone (HCon ou $\mathrm{HCiF}$ ) que seria utilizado nos experimentos, o mesmo era acoplado à unidade experimental. A suspensão de rocha fosfática utilizada durante os ensaios experimentais foi preparada com uma concentração volumétrica de material particulado de $1 \%$.

Neste estudo adotaram-se as seguintes condições de queda de pressão: $88,118,147$ e $177 \mathrm{kPa}$, à temperatura de 293K.

Iniciado o experimento, eram medidas as vazões de alimentação, underflow, overflow e filtrado para os hidrociclones, sendo que a vazão de alimentação era verificada através do medidor de vazão e as demais eram obtidas através de técnicas gravimétricas. A concentração mássica de cada corrente (alimentação, overflow e underflow) foi determinada pela coleta de pequenas amostras seguidas por um teste de estufa por 24 horas. Para a realização da análise da distribuição granulométrica foram retiradas alíquotas das correntes de alimentação e underflow.

As medidas experimentais obtidas segundo os procedimentos anteriormente descritos para vazões mássicas, concentrações mássicas e distribuição granulométrica eram manipuladas com intuito de verificar as repostas das variáveis que estão associadas aos hidrociclones. As variáveis calculadas eram Vazões Volumétricas $(Q)$, Número de Euler $(E u)$, Razão de Líquido $\left(R_{L}\right)$, Eficiência Total $(\eta)$.

\subsection{Determinação das Curvas de Eficiência}

Além da comparação da performance entre o hidrociclone convencional e o filtrante, foram determinadas neste trabalho, curvas de eficiência total para o hidrociclone cilíndrico filtrante através da metodologia descrita por Massarani (2002). Essa metodologia permite o levantamento teórico das curvas de eficiência total para outras distribuições granulométricas (genéricas) a partir de uma distribuição granulométrica específica e das condições geométricas e operacionais do hidrociclone.

\section{RESULTADOS}

Os resultados obtidos mostraram que a vazão de filtrado representou no máximo $0,1 \%$ da vazão de alimentação. Apesar disso, verificou-se que, mesmo ínfima, a presença dessa nova corrente foi capaz de modificar o funcionamento do hidrociclone cilíndrico filtrante.

A Figura 3 mostra a relação entre a vazão volumétrica de alimentação e a queda de pressão para os hidrociclones convencional 
e filtrante. Analisando os resultados experimentais percebeu-se que um aumento na queda de pressão majorou a vazão de alimentação dos separadores independente do hidrociclone e da permeabilidade utilizados. Isso se explica pelo fato de mais energia estar sendo fornecida para o escoamento do fluido.

Em se tratando somente dos hidrociclones filtrantes verificou-se que a vazão de alimentação diminuiu com o incremento da permeabilidade. Acredita-se que essa diminuição ocorreu, pois, a corrente de filtrado pode ter alterado a relação entre as componentes da velocidade do fluido. Quando ocorreram as maiores taxas de filtração, a componente radial do fluido foi aumentada à custo das demais componentes, diminuindo o movimento espiralado no interior do hidrociclone, que por sua vez, está relacionado com a capacidade de alimentação do hidrociclone (VIEIRA, 2006).

Figura 3 - Vazão de alimentação em função da queda de pressão para o hidrociclone HCon e para os hidrociclones $\mathrm{HCiF}$

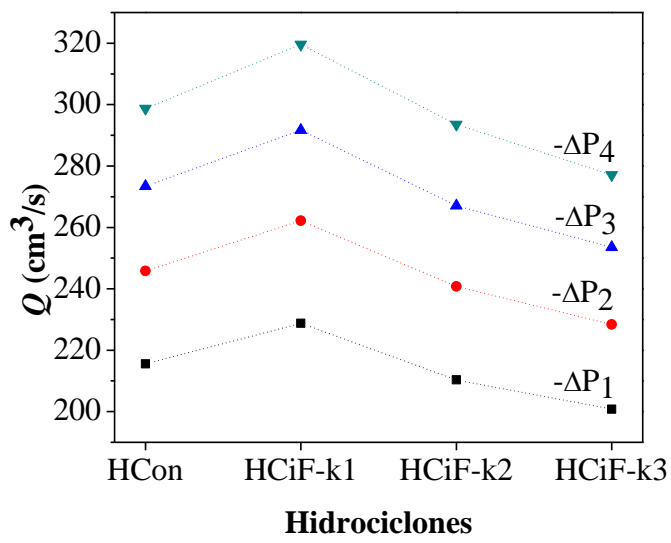

Analisando a Figura 3 observou-se que a vazão de alimentação do hidrociclone cilíndrico filtrante foi maior ou menor do que a do hidrociclone convencional a depender da permeabilidade utilizada. A vazão de alimentação chegou a seu valor máximo quando a menor permeabilidade foi utilizada (em média $7 \%$ maior do que para 0 hidrociclone HCon) e a seu valor mínimo quando utilizou-se a permeabilidade $\mathrm{k} 3$ (em média $7 \%$ menor do que para o hidrociclone HCon).

É sabido que o número de Euler está ligado à relação entre a queda de pressão e a vazão de alimentação do hidrociclone, logo, é de se esperar que um aumento na vazão de alimentação tenha ocasionado um decréscimo nesse número adimensional. A Figura 4 apresenta os valores médios obtidos para o número de Euler para os hidrociclones convencional e filtrantes estudados. Ao analisar os resultados verificou-se que, quanto maior a permeabilidade maior foi o número de Euler.

Figura 4 - Número de Euler para o hidrociclone $\mathrm{HCon}$ e para os hidrociclones $\mathrm{HCiF}$

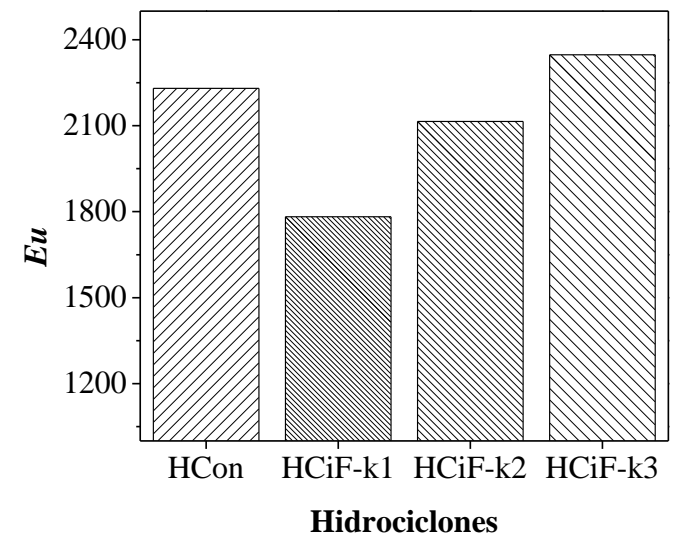

Observando a Figura 4, percebeu-se que, assim como foi verificado para a vazão de alimentação, o número de Euler para os hidrociclones $\mathrm{HCiF}$ foi menor ou maior do que para o hidrociclone HCon a depender da permeabilidade utilizada. O consumo dos hidrociclones HCiF-k1 e HCiF-k2 foram $20 \%$ e $4 \%$, respectivamente, menores do que o do hidrociclone HCon. Já o hidrociclone HCiF$\mathrm{k} 3$ teve o seu gasto energético aumentado $6 \%$, em média, em relação ao hidrociclone convencional.

A Figura 5 mostra os resultados para a razão de líquido média dos hidrociclones 
convencional e filtrantes. Os resultados obtidos mostraram que esta variável permaneceu praticamente constante com a variação da queda de pressão. Analisando a influência da permeabilidade, percebeu-se que um incremento na mesma provocou decréscimos na razão de líquido. Tem-se então que um aumento na permeabilidade, de $\mathrm{k} 1$ para k2, diminuiu a razão de líquido cerca de $14 \%$, em média, sendo que outro aumento na permeabilidade, de k2 para k3, levou a decréscimos de $25 \%$, em média, na resposta em questão. Acredita-se que a corrente adicional de filtrado causou uma interferência na relação entre os volumes dos vórtices externo e interno, ocasionando uma modificação na fração de líquido que foi enviada para as correntes de underflow e overflow, diminuindo a quantidade de líquido descarregada no underflow.

Figura 5 - Razão de Líquido para o hidrociclone HCon e para os hidrociclones $\mathrm{HCiF}$

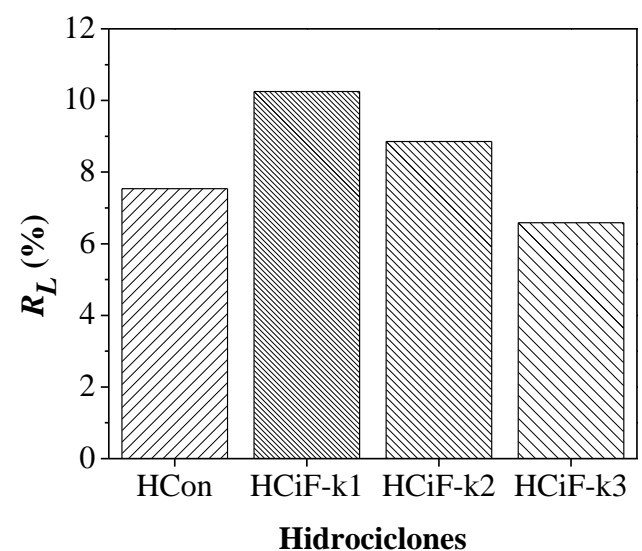

Ao comparar a razão de líquido dos hidrociclones HCon e $\mathrm{HCiF}$, verificou-se que apenas o hidrociclone cilíndrico filtrante de maior permeabilidade teve o valor dessa resposta menor do o hidrociclone HCon. Sendo que o hidrociclone HCiF-k1 foi o que teve o aumento mais acentuado, cerca de $36 \%$ em relação ao hidrociclone convencional.

A Figura 6 apresenta a eficiência total dos separadores convencional e cilíndrico filtrantes. Analisando somente os hidrociclones cilíndrico filtrantes verificou-se que a permeabilidade do meio filtrante não provocou alterações significativas na eficiência total desses equipamentos. A variação na eficiência total foi menor do que $1 \%$ quando se modificou a permeabilidade da parede de k1 para k2 e de k2 para k3.

Figura 6 - Eficiência Total para o hidrociclone $\mathrm{HCon}$ e para os hidrociclones $\mathrm{HCiF}$

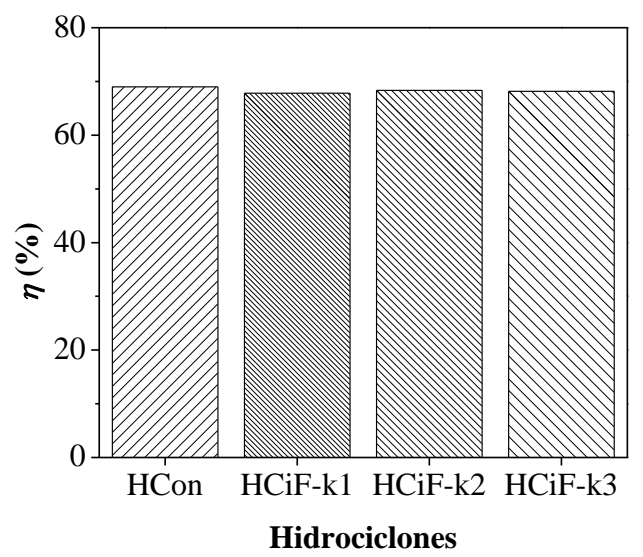

É interessante observar que, mesmo a razão de líquido tendo sido diminuída com o aumento de permeabilidade (comportamento indesejado para a separação), tal fenômeno foi praticamente irrelevante sobre a eficiência total, que mostrou não depender da taxa de filtração utilizada. Essa indiferença deve ter ocorrido provavelmente porque, ao mesmo tempo em que diminuía a razão de líquido, certamente houve também um aumento na velocidade radial do fluido em direção a parede porosa, que é um comportamento benéfico para a separação. Desta forma, os efeitos das velocidades axial e radial foram combinados, de modo que um compensou a ação do outro, mantendo a eficiência total no mesmo nível.

Ainda observando a Figura 6, percebeuse que a eficiência total não foi modificada devido ao posicionamento do meio filtrante, pois a eficiência do hidrociclone convencional foi no máximo $1,3 \%$ maior do que as 
eficiências dos hidrociclones filtrantes (esse valor está dentro da incerteza da medida).

Figura 7 - Curvas de Eficiência Total do hidrociclone cilíndrico filtrante para os diâmetros de underflow de (a) 3, (b) 4 e (c) $5 \mathrm{~mm}$ em função de diferentes distribuições granulométricas.

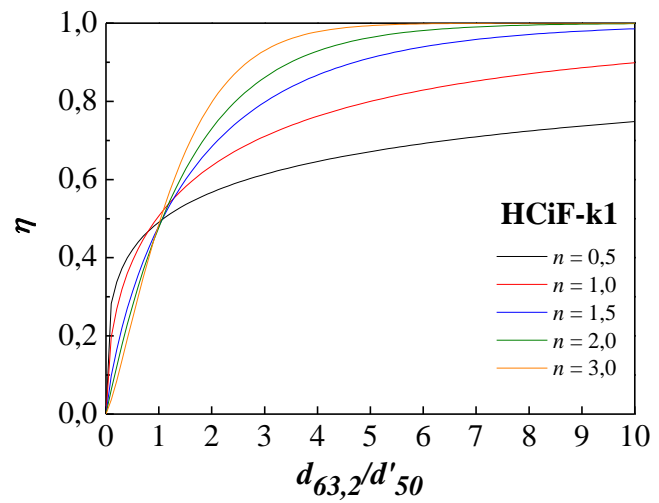

(a)

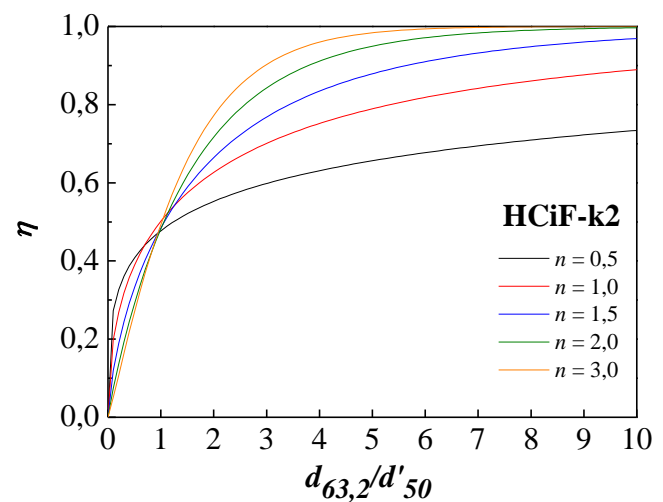

(b)

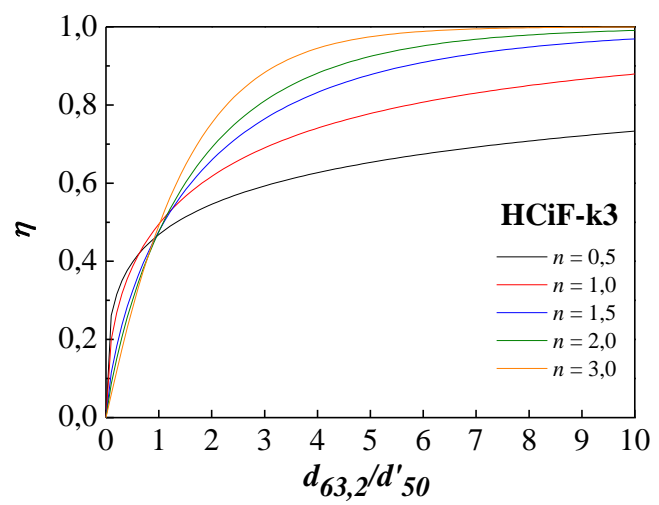

(c)

A fim de que os hidrociclones $\mathrm{HCiF}$ pudessem ser utilizados por possíveis interessados, levantaram-se as curvas de eficiência total, que estão apresentadas nas Figuras 7(a), 7(b) e 7(c) referentes aos hidrociclones HCiF-k1, HCiF-k2 e HCiF-k3, respectivamente.

As curvas de eficiência da Figura 7 mostraram que o desempenho do hidrociclone cilíndrico filtrante é extremamente dependente da granulometria da fase particulada que lhe é alimentada. Os resultados apresentados nos gráficos sugerem que quanto maior o diâmetro da partícula maior é a eficiência total do separador filtrante.

\section{CONCLUSÕES}

Considerando o objetivo deste trabalho, que foi avaliar a influência da permeabilidade no desempenho do hidrociclone $\mathrm{HCiF}$ e fazer uma comparação desse separador com o hidrociclone HCon, foi possível constatar que:

- A vazão de alimentação dos hidrociclones filtrantes diminuiu ou aumentou em relação ao hidrociclone convencional a depender da permeabilidade do meio poroso. Então, se o principal interesse for um equipamento com elevada capacidade de processamento, permeabilidades menores ou iguais a $\mathrm{k} 1\left(1,91.10^{-16} \mathrm{~m}^{2}\right)$ são preferíveis.

- O número de Euler para o hidrociclone filtrante diminuiu ou aumentou em comparação com o hidrociclone convencional de acordo com a permeabilidade empregada. Assim o hidrociclone HCiF-k1 seria o mais viável, analisando somente pela óptica do consumo energético.

- Incrementos na permeabilidade do meio filtrante provocaram uma queda na razão de líquido dos hidrociclones $\mathrm{HCiF}$. Foi observado ainda que a retirada de filtrado fez com que os equipamentos filtrantes, em relação ao separador convencional, tivessem acréscimos ou decréscimos em sua razão de líquido. 
- A eficiência total dos separadores filtrantes não apresentou modificações significativas frente ao valor obtido para o hidrociclone convencional, pois as diferenças entre as eficiências dos hidrociclones estavam dentro da incerteza da medida. Constatou-se também que a modificação da permeabilidade da parede filtrante não alterou a eficiência total de separação dos hidrociclones $\mathrm{HCiF}$.

\section{NOMENCLATURA}

$d_{50}^{\prime} \quad$ Diâmetro de corte reduzido [mm]

$d_{63,2}$ Parâmetro ajustado do modelo RRB [mm]

$D_{c} \quad$ Diâmetro da parte cilíndrica do hidrociclone [mm]

$D_{i} \quad$ Diâmetro da alimentação do hidrociclone [mm]

$D_{o} \quad$ Diâmetro do duto de overflow do hidrociclone [mm]

$d_{p} \quad$ Diâmetro da partícula [mm]

$D_{u} \quad$ Diâmetro do duto de underflow do hidrociclone [mm]

Eu Número de Euler

$h \quad$ Altura da parte cilíndrica [mm]

$H \quad$ Altura da parte cônica [mm]

$\ell \quad$ Comprimento do vortex finder [mm]

$L \quad$ Comprimento total do hidrociclone [mm]

n Parâmetro ajustado do modelo RRB

$Q \quad$ Vazão volumétrica da corrente de alimentação do hidrociclone $\left[\mathrm{cm}^{3} / \mathrm{s}\right.$ ]

$R_{L} \quad$ Razão de líquido

$X \quad$ Fração mássica de partículas iguais ou menores do que $d_{p}$ na corrente de alimentação

$-\triangle P \quad$ Queda de pressão entre a alimentação e overflow do hidrociclone [kPa]

$\eta \quad$ Eficiência total

$\theta \quad$ Ângulo do tronco de cone $\left[{ }^{\circ}\right]$

\section{REFERÊNCIAS}

CARRISSO, R. C. C.; CORREIA, J. C. G. Classificação e Peneiramento. In: Luz, A. B.; Sampaio, J. S; Almeoida, S. L. M. Tratamento de Minérios. $4^{\mathrm{a}}$ ed. Rio de Janeiro: CETEM/MCT, 2004. p.197-237.

CHINE, B.; CONCHA, F. Flow patterns in conical and cylindrical hydrocyclones. Chemical Engineering Journal, v.80, p.267273, 2000.

DONG, L.; FAN, M.; YANG, H. Separation performance of a cyclone column separator with complicated positive and negative cones. International Journal of Mineral Processing, v.122, p.43-46, 2013.

GHODRAT, M.; KUANG, S. B.; YU, A. B.; VINCE, A.; BARNETT, G. D.; BARNETT, P. J. Numerical analysis of hydrocyclones with different conical section designs. Minerals Engineering, v.62, p.74-84, 2014.

HE, F.; ZHANG, Y.; WANG, J.; YANG, Q.; WANG, H.; TAN, Y. Flow Patterns in MiniHydrocyclones with Different Vortex Finder Depths. Chemical Engineering Technology, v.36, p.1935-1942, 2013.

MASSARANI, G. Fluidodinâmica em Sistemas Particulados. $2^{\mathrm{a}}$ ed. Rio de Janeiro: E-Papers Serviços Editoriais, 2002.

ROCHA, S. M. S.; VIEIRA, L. G. M. Experimental determination of the permeability of non-planar porous structures during operation of equipment in own industrial unit. Materials Science Forum, v.727, p.1836-1841, 2012.

SILVA, D. O.; VIEIRA, L. G. M.; BARROZO, M. A. S. Optimization of Design and Performance of Solid-Liquid Separators: A Thickener Hydrocyclone, Chemical Engineering Technology, v.38, p.319-326, 2015. 
SILVA, D. O.; VIEIRA, L. G. M.; LOBATO, F. S.; BARROZO, M. A. S. Optimization of the design and performance of hydrocyclones by Differential Evolution technique. Chemical Engineering and Processing: Process Intensification, v.1, p.1-12, 2012.

SOUZA, F. J.; VIEIRA, L. G. M.; BARROZO, M. A. S.; DAMASCENO, J. J. R. Analysis of the Influence of the Filtering Medium on the Behaviour of the Filtering Hydrocyclone. Powder Technology, v.107, p.259-267, 2000.

SRIPRIYA， R.; KAULASKAR， M. D.; CHAKRABORTY, S.; MEIKAP, B. C. Studies on the performance of a hydrocyclone and modeling for flow characterization in presence and absence of air core. Chemical Engineering Science, v.62, n.22, p.63916402, 2007.

SVAROVSKY, L. Hydrocyclones. Eastbourne, UK: Holt, Rinehart \& Winston, 1984.

SVAROVSKY, L. Solid-Liquid Separation. $4^{\mathrm{a}}$ Ed. Linacre House, Jordan Hill, Oxford OX2 8DP: Butterworth-Heinemann, 2000.

VIEIRA, L. G. M. Otimização dos Processos de Separação em Hidrociclones Filtrantes. 2006. 297p. Tese (Doutorado em Engenharia Química) -Faculdade de Engenharia Química, Universidade Federal de Uberlândia, Uberlândia, 2006.

VIEIRA, L.G.M.; DAMASCENO, J. J. R.; BARROZO, M. A. S. Improvement of hydrocyclone separation performance by incorporating a conical filtering wall. Chemical Engineering and Process, v.49, p.460-467, 2010.

VIEIRA, L. G. M.; SILVA JR, C. A.; DAMASCENO, J. J. R.; BARROZO, M. A.
S. A study of the fluid dynamic behaviour of filtering hydrocyclones. Separation and Purification Technology, v.58, p.282-287, 2007.

VIEIRA, L.G.M.; SILVÉRIO, B.C.; DAMASCENO, J.J.R.; BARROZO, M.A.S. Performance of hydrocyclones with different geometries. The Canadian Journal of Chemical Engineering, v.89, p.655-662, 2011.

\section{AGRADECIMENTOS}

Os autores agradecem ao $\mathrm{CNPq}$, à CAPES e à FAPEMIG pelo apoio financeiro durante a realização do trabalho $\mathrm{e}$ ao Laboratório de Separação e Energias Renováveis (LASER) da FEQUI/UFU. 